\title{
A SEARCH FOR LONG-PERIOD CEPHEIDS IN ASSOCIATIONS
}

\author{
Sidney van den Bergh \\ Dominion Astrophysical Observatory \\ Herzberg Institute of Astrophysics \\ 5071 W. Saanich Road, Victoria, B.C. V8X 4M6, Canada
}

Abstract. Skeleton photoelectric sequences and plates in $U$, $B$ and $V$ have been obtained for fields surrounding all known Cepheids with $P \geqslant 12$ days in the Southern Milky Way. The study of 14 of these fields has been completed and is discussed in this paper.

Cepheid variables remain the most important calibrators of the extra-galactic distance scale. Unfortunately the Cepheids that are presently known in open clusters are intrinsically fainter, and have shorter periods, than do those that can be detected in Galaxies beyond the Local Group. It is therefore important to calibrate long-period Cepheids, similar to those that we observe in distant galaxies, in our own galaxy. At Cerro Tololo I have therefore obtained skeleton UBV sequences around all Population I Cepheids with $P \geqslant 12$ days, that are located along the Southern Milky Way. Plates of fields surrounding these same Cepheids have been obtained in ultraviolet, blue and yellow light by Brosterhus and Younger with the $1-\mathrm{m}$ Swope telescope on Las Campanas. Alcaino and Turner have contributed to the measurements (typically $300-800$ stars in each field) and to the reduction of these data.

To date observations of the following Cepheids have been reduced:

$\begin{array}{rlll}\text { U Car 38.8 } & \text { QY Cen } 17.8 & \text { AA Nor } 12.3 \\ \text { EZ Vel 34.5 } & \text { XZ Car 16.5 } & \text { KK Cen } 12.2 \\ \text { VZ Pup 23.2 } & \text { YZ Car 16.6 } & \text { VX Cru } 12.2 \\ \text { WZ Car 23.0 } & \text { SV Ve1 } 14.1 & \text { UU Mus } 11.6 \\ \text { CT Car 18.1 } & \text { OO Cen } 12.9 & \end{array}$

Results on WZ Car, YZ Car, KK Cen and OO Cen have been published by van den Bergh, Brosterhus and Alcaino (1982). Those on CT Car, UU Mus, VZ Pup, SV Vel and EZ Vel by van den Bergh, Younger, Brosterhus and Alcaino (1983) and those on U Car, XZ Car, QY Cen, VX Cru and AA Nor by van den Bergh, Younger and Turner (1984). The results obtained to date are summarized below:

U Carinae. This Cepheid is located $\sim 1: 5$ east of the center of the $\bar{n}$ Car nebula. The photometry suggests that $U$ Car is somewhat closer to us than the $n$ Car complex. 
EZ Velorum. This object is not embedded in a major association that is young enough to contain $0-B 1$ stars. The present observations do not extend faint enough to detect an association containing main-sequence stars with spectral types later than Bl.

VZ Puppis. Somewhat surprisingly the low-absorption field surrounding this long-period Cepheid contains no stars with $B-V<0.0$ or $U-B<-0.50$. The absence of such stars is puzzling because VZ Pup (if it is a Cepheid of Population I) has an age of on $1 \mathrm{y} \sim 2 \times 10^{7}$ years.

WZ Carinae. There is no evidence to suggest that WZ Car is embedded in a major association.

CT Carinae. The UBV colors of stars in this field may be interpreted in two ways. Either CT Car is embedded in a rich association of distant B stars or it is projected on a concentration of foreground F-type stars. Spectroscopy will be required to distinguish between these two alternatives.

QY Centauri. The situation in this field is confused. It is not yet clear if the early-type stars in this area are at the same distance as the Cepheid.

$X Z$ Carinae. This Cepheid lies at a distance comparable to that of the rich Car OBl complex. Twelve open clusters 1 ie within $1^{\circ}$ of this variable. The closest of these is the unstudied cluster Ruprecht 93.

YZ Carinae. The color-color diagram shows no evidence for significant numbers of reddened $O B$ stars in this field.

SV Velorum. Two B-type stars with reddening values similar to the Cepheid are located in this field.

DO Centauri. Three stars in this field have UBV colors indicating that they are $O B$ stars with reddenings similar to that of the Cepheid. A deeper study of this field would be desirable.

AA Normae. This object is probably a Cepheid of Population II.

KK Centauri. No objects with the colors of reddened OB stars are seen in this field.

VX Crucis. This Cepheid is not embedded in an association containing very early-type stars. Deeper photometry (particularly in U) would be required to detect late $B$ stars in this field.

UU Muscae. The color-magnitude and color-color diagrams of the field surrounding this Cepheid show no evidence for a concentration of blue stars that might be associated with UU Mus.

The results that are summarized above are, in some ways, disappointing. Reasons for this are probably that: 
1. Most long-period Cepheids are not situated in the cores of rich associations.

2. In some poor associations the surface density of $O B$ stars may be too low for significant numbers of such objects to be situated within $10^{\prime}$ of a Cepheid.

3. Associations containing mainly late B-type stars may, in a few cases, have been missed because our photometry did not go deep enough in some reddened fields.

We hope to be able to continue publishing the results of this search for long-period Cepheids in associations at the rate of about half a dozen fields per year.

\section{REFERENCES}

van den Bergh, S., Brosterhus, E.B.F. \& Alcaino, G. (1982). Ap.J.Suppl., $50,529$.

van den Bergh, S., Younger, P.F., Brosterhus, E.B.F. \& Alcaino, G. (1983). Ap.J.Supp1., 53, 765.

van den Bergh, S., Younger, P.F. \& Turner, D.G. (1984). In preparation. 